\title{
Discussion of "The Structure-Soil-Structure Interaction Effects on the Response of the Neighbouring Frame Structures"
}

\author{
Huai-feng Wang ${ }^{\mathrm{a}^{*}}$ \\ aSchool of Civil Engineering and Architecture, Xiamen University of Technology, Xiamen 361000, China. Email: whf_tj@163.com \\ * Corresponding author \\ http://dx.doi.org/10.1590/1679-78256041
}

\begin{abstract}
This discussion raises a few comments and questions on the paper by Mehmet ADA and Yusuf AYVAZ [ADA, M. and Y. AYVAZ, The Structure-Soil-Structure Interaction Effects on the Response of the Neighbouring Frame Structures. Latin American Journal of Solids and Structures, 2019. 16(8)].
\end{abstract}

Keywords

Dynamic Analysis; Soil-Structure Interaction; Structure-Soil-Structure Interaction; Seismic Response; Finite Element Method

\section{INTRODUCTION}

The discussed paper (Ada and Ayvaz, 2019) presented the structure-soil-structure interaction (SSSI) effects on the response of the neighboring frame structures. The discusser appreciates the authors' meaningful work. Some findings are interesting to the discusser and are worthy of further discussion.

\section{DISCUSSION AND QUESTIONS}

1. Rayleigh damping was introduced for modeling. As a result, it is known that two frequencies should be assigned for the calculation of Rayleigh coefficients through Eq. (2) in this paper. The fundamental frequency and its odd integer multiplier were used in the paper to calculate the Rayleigh coefficients of the soil medium. The odd integer was calculated by rounding up the dominant frequency ratio of the input motion (the highest frequency value with a substantial Fourier amplitude) to the fundamental frequency of the soil. To the best of the author's knowledge, this method was proposed by Idriss (Idriss and Lysmer et al., 1973), based on a one-dimensional soil model, and its applicability to a three-dimensional soil-structure model should be further verified.

More importantly, Lou (Lou and Shao, 2013) verified that, for a system with a fundamental frequency much lower than the dominant frequency of the input motion, frequencies should be carefully selected for the calculation of Rayleigh coefficients; otherwise, the dynamic response will be exaggerated. In this paper, the frequencies for the calculation of Rayleigh coefficients are not listed distinctly, and the numerical error due to Rayleigh damping is not analyzed. However, for soil type 1 (shear-wave velocity=150 m/s), the fundamental frequency of the soil is obviously lower than the dominant frequency of the input motion, which is closer to the second-mode frequency of the structure with 12 stories along the shaking direction (mode 4 and mode 5 in this paper). Thus, it is necessary to check whether the dynamic response of the second mode of the structure with 12 stories was overexaggerated and whether the dynamic response of the third mode of the structure with 12 stories was overcompressed. In our study, for a widespread frame, SSSI mainly arises around the first several modes of the structure along the shaking direction. If the dynamic response of the second mode was exaggerated too much or if the dynamic response of the third mode was compressed too much, the latter analysis of SSSI in the paper may be meaningless. 
Moreover, many studies (Padron and Aznarez et al., 2009; Alexander and Ibraim et al., 2013; Wang and Lou et al., 2017; Vicencio and Alexander, 2019) have shown the strong frequency dependency of SSSI. Any numerical error due to Rayleigh damping should be analyzed inevitably for the frequency dependency of Rayleigh damping.

2. The inadequacy of the finite element size may cause numerical damping. As mentioned in this paper, the desired number of elements per wavelength ( $\mathrm{n}$ in Eq. (3) in this paper) can be chosen from 5 to 8 , which usually leads to satisfactory results. However, this conclusion is applicable to the soil-structure system but not to the structure-soil-structure system. Based on our work, the desired number of elements per wavelength should be range from 8 to 12 , since SSSI itself is very susceptible to numerical error. In fig. 3 of this paper, the numerical validation of the assigned finite element size is presented for soil type 1 (shear-wave velocity=150 m/s). Although the Fourier amplitudes for finite element sizes of $2 \mathrm{~m}$ and $1 \mathrm{~m}$ are almost consistent in the low-frequency range and only slightly inconsistent in the high-frequency range, the reader should note that this difference is reflected at the locations $16 \mathrm{~m}$ and $24 \mathrm{~m}$ away from the top center of the soil domain. Based on wave motion theories (Achenbach, 1999), the energy dissipation of high-frequency waves is faster than that of low-frequency waves during propagation. Thus, the faster energy dissipation of high-frequency waves and the numerical damping of the mesh may result in consistency for high-frequency waves, which can be verified indirectly in fig. 3. For finite element sizes of $4 \mathrm{~m}$ and $8 \mathrm{~m}$, the error of the Fourier amplitudes at $24 \mathrm{~m}$ away from the top center of the soil domain is less than that at $16 \mathrm{~m}$. Since the considered clear distance reaches $2 \mathrm{~m}$, the Fourier amplitudes at $2 \mathrm{~m}$ away from the top center of the soil domain should be checked further.

3. In this paper, the maximum acceleration value of the structure was employed to analyze the influence of SSSI. Without a doubt, the maximum indicated the maximum value of the acceleration time history. However, did it indicate the maximum value of the acceleration of each floor? If so, the number or position of the floor with the maximum acceleration should be explicated. In our study, the influence of SSSI on the acceleration of each floor is not exactly the same. The influences on the high and low floors are larger than those on the middle floors.

4. In this paper, the considered clear distances are $2 \mathrm{~m}, 4 \mathrm{~m}, 8 \mathrm{~m}, 16 \mathrm{~m}, 32 \mathrm{~m}$ and $48 \mathrm{~m}$. In some numerical calculations, with an increase in the clear distance, the influence of SSSI on the dynamic response of the structure did not decrease and did not tend to zero, as shown in fig. 6(e), fig. 6(f), fig. 7(a), fig. 7(c), fig. 8(b), fig. 8(f), fig. 9(b), fig. 9(e) and so on. This is very counterintuitive. The authors of the discussed paper concluded that the resonance state of 12 -storey structure results in the increase or the constant. However, the counterintuitive phenomenon also occurred in the system of soil and structures with 3 stories and 6 stories. The author postulates that one reason may be the phase of the scattered wave from the vibrating structure. Further research should be conducted.

5. This paper concluded that, in the case of the aligned layouts, the peak variation in the structure is observed at close distances; however, the peak variation is observed in the mid-distance range for the unaligned layouts. The former phenomenon can be easy to understand, but the latter phenomenon is puzzling. Perhaps this can be clarified by the authors of the discussed paper.

\section{Acknowledgment}

The author would like to gratefully acknowledge the support from National Natural Science Foundation of China under Grant No. 51608462.

Editor: Marcílio Alves.

\section{References}

Achenbach, J. D. (1999). Wave Propagation in Elastic Solids. Amsterdam, Netherlands.

Ada, M. and Y. Ayvaz (2019). The Structure-Soil-Structure Interaction Effects on the Response of the Neighbouring Frame Structures. Latin American Journal of Solids and Structures 16 (8).

Alexander, N. A. and E. Ibraim, et al. (2013). A simple discrete model for interaction of adjacent buildings during earth-quakes. Computers \& Structures 124 (SI): 1-10.

Idriss, I. M. and J. Lysmer, et al. (1973). QUAD4: A Computer Program for Evaluating the Seismic Response of Soil Structures by Variable Damping Finite Element Procedures, Berkeley: University of California.

Padron, L. A. and J. J. Aznarez, et al. (2009). Dynamic structure-soil-structure interaction between nearby piled buildings under seismic excitation by BEM-FEM model. Soil dynamics and earthquake engineering 9 (6): 1084-1096. 
Vicencio, F. and N. A. Alexander (2019). Dynamic Structure-Soil-Structure Interaction in unsymmetrical plan buildings due to seismic excitation. Soil Dynamics and Earthquake Engineering 127: 105817.

Wang, H. and M. Lou, et al. (2017). Influence of presence of adjacent surface structure on seismic response of underground structure. Soil Dynamics and Earthquake Engineering 100 (09): 131-143.

Lou, M. and X. Shao (2013). Discussion on modeling issues of Rayleigh damping matrix in soil layers with deep deposit. Chinese Journal of Geotechnical Engineering 35 (07): 1272-1279. (in Chinese) 